\title{
Nonlinear Dynamics of Congestive Heart Failure
}

\author{
A. Bernjak ${ }^{a}$, P.B.M. Clarkson ${ }^{b}$, P.V.E. McClintock ${ }^{c}$, A. Stefanovska ${ }^{a, c}$ \\ ${ }^{a}$ Faculty of Electrical Engineering, University of Ljubljana, Slovenia \\ ${ }^{b}$ Cardiology Department, Royal Lancaster Infirmary, LA1 4RP, UK \\ ${ }^{c}$ Physics Department, Lancaster University, LA1 4YB, UK
}

\begin{abstract}
Preliminary results are reported from a research project analysing congestive heart failure in terms a stochastic coupled-oscillator model of the cardiovascular system. Measurements of blood flow by laser Doppler flowmetry (LDF) have been processed by use of the wavelet transform to separate its oscillatory components, which number at least five. Particular attention was concentrated on the frequency content near $0.01 \mathrm{~Hz}$, which is known to be associated with endothelial function. The LDF was carried out in conjunction with iontophoretically administered acetylcholine $(\mathrm{ACh})$ and sodium nitroprusside (SNP) in order to evaluate endothelial reactivity. Measurements were made on 17 congestive heart failure (CHF) patients (a) on first diagnosis, and (b) again several weeks later after their treatment with a $\beta$-blocker had been stabilised. The results of these two sets of measurements are being compared with each other, and with data from an age and sex-matched group of healthy controls. It is confirmed that endothelial reactivity is reduced in CHF patients, as compared to healthy controls, and it is found that one effect of the $\beta$-blocker is to ameliorate the loss of endothelial function in CHF. The implications of these results are discussed.
\end{abstract}

Keywords: noisy oscillators, coupled oscillators, blood flow, heart rate variability, heart failure, iontophoresis, vasodilation, wavelet transform, endothelium,

\section{INTRODUCTION}

The perception of the cardiovascular system as a set of noisy coupled oscillators ${ }^{1}$ has opened new possibilities in the analysis of signals derived from it, e.g. of the cardiac and respiratory functions, blood flow and blood pressure. At least five oscillatory processes can be identified. Their relative amplitudes, and the strengths of the interactions between them, have obvious potential to illuminate the underlying physiological processes giving rise to the oscillations, and hence to give early warning of abnormalities perhaps corresponding to the onset of disease.

We describe below an analysis of changes in oscillatory behaviour associated with congestive heart failure. We measure patients' cutaneous blood flow, and assess the effects of two different iontophoretically delivered vasodilators: acetylcholine $(\mathrm{ACh})$ and sodium nitroprusside (SNP). The vasodilating actions of ACh and SNP are respectively endothelium-dependent and endothelium-independent, so that comparison of the increases in blood flow oscillatory amplitudes effected by each enables an assessment of endothelial reactivity. The wavelet transform is used to separate the oscillatory components at different frequencies and, in particular, to assess the relative energy content at $\sim 0.01 \mathrm{~Hz}$ which is known ${ }^{2}$ to be associated with endothelial function. Measurements have been completed on 17 congestive heart failure (CHF) patients, for each of them on two separate separate occasions: (a) on first diagnosis, prior to the initiation of treatment; and (b) several weeks later, after stabilisation on treatment with a $\beta$-blocker. In each case, cutaneous blood flow was measured with and without the ACh and SNP. The ECG, respiration, and blood pressure were simultaneously recorded. Identical measurements have also been made on a control group of age-matched healthy subjects.

Treatment of CHF is now orientated towards a long term reparative strategy that aims favourably to alter the biological properties of the failing heart. ${ }^{3}$ This is dramatically illustrated by recent successes in the treatment of mid- to moderate-CHF with $\beta$-adrenergic blocking agents. ${ }^{4,5} \beta$-blockers have been shown to reduce heart

Correspondence to: p.v.e.mcclintock@lancaster.ac.uk 


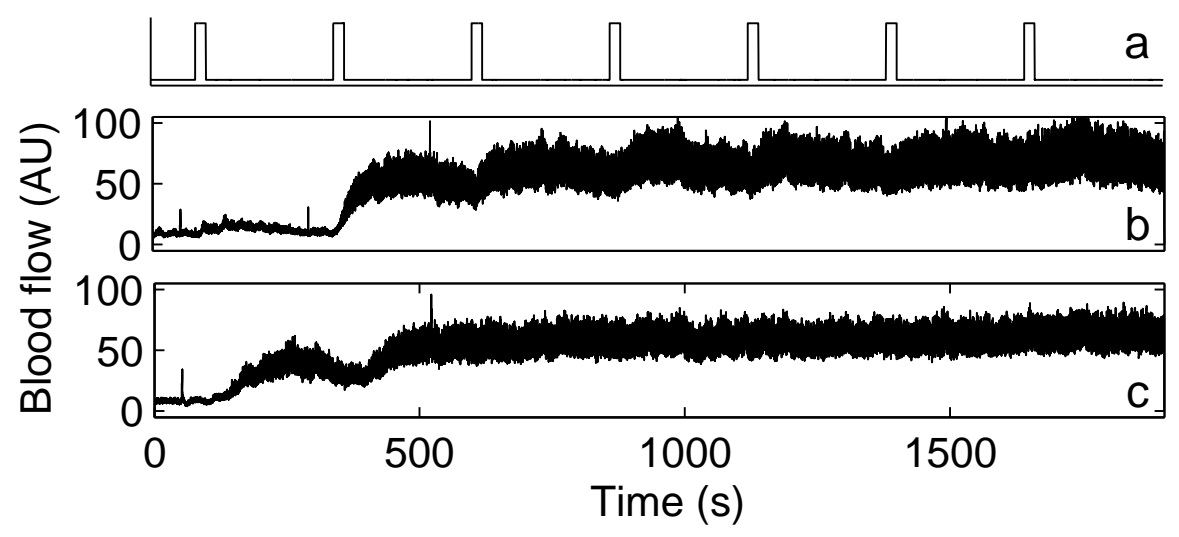

Figure 1. Simultaneously measured LDF signals showing how blood flow changes in response to iontophoresis of the two vasodilators. a, Timing of the $100 \mu \mathrm{A}$ iontophoresis current pulses; b, blood flow stimulated by ACh; c, blood flow stimulated by SNP.

rate, decrease the firing rate of ectopic pacemakers, slow conduction and increase the refractory period of the atroventricular (AV) node. ${ }^{5}$ This results in a long-term reduction in total mortality, cardiovascular mortality, sudden cardiac death, and death following progression of the CHF through the functional New York Heart Association (NYHA) Classes II-IV. At the same time, CHF is characterised by peripheral vascular problems including systemic vasoconstriction ${ }^{6}$ and reduced regional blood flow, ${ }^{7}$ where the effect of $\beta$-blockers is not known. The endothelium plays a pivotal role in regulating blood flow by releasing relaxing and constricting factors, a role that has been shown to be impaired in $\mathrm{CHF}^{8-10}$ Various technologies are available to evaluate endothelial function, like plethysmography or laser-Doppler flowmetry (LDF) that monitor vasodilatory responses to the administration of an endothelial-dependent vasodilator such as acetylcholine (ACh). A decreased response to ACh but not to the endothelial-independent vasodilator sodium nitroprusside (SNP) is considered evidence of endothelial dysfunction. Besides basal differences in responses to these two substances, one can study oscillatory components in the blood flow. ${ }^{11-13}$ Using this technique, we further hypothesise that the difference in endothelial reactivity of the $\mathrm{CHF}$ group and an age-matched healthy control (HC) group will be manifested in particular oscillatory components. The central hypothesis motivating our study is that, besides regularisation of the heart rhythm, $\beta$-blockers have a beneficial effect on endothelial responsiveness in CHF.

\section{EXPERIMENTAL METHODS}

Patients for the CHF group were recruited from the Morecombe Bay heart failure cardiology clinic register. Inclusion criteria were that they should be in Class III or IV of the NYHA classification. Exclusion criteria included: hypertension, diabetes, vascular disease, or any vascular medications. Healthy control subjects were recruited from the local community and screened by history and examination, including ECG. After the CHF patients had been treated with Bisoprolol (a $\beta$-blocker), and their dosage had been titrated up to their maximum tolerated, they were remeasured, now as the $\beta-\mathrm{CHF}$ subject group. All participants gave their informed consent in writing. The investigation conformed with the principles outlined in the Declaration of Helsinki and was approved by the Local Ethics Committee of the Morecombe Bay Hospitals Trust. Titration.

We asked subjects to lie down on a bed and relax for 15 minutes prior to making continuous, simultaneous, measurements of: peripheral blood flow by laser Doppler flowmetry (LDF); heart rate, using a conventional 3-lead ECG; respiration, using a Biopac respiratory effort transducer placed around the thorax; blood pressure, using a piezo-electric pulse plethysmograph placed on a finger; and temperature, using a sensitive fast-acting thermometer placed on the skin. The temperature of the room was maintained at $21 \pm 2{ }^{\circ} \mathrm{C}$. For the present study, only the blood flow measurements were used directly but the other recordings helped us to guard against the occurrence of systematic errors during the period of measurement. Recording lasted for 30 minutes, chosen as a compromise between making the time series as long as possible, in order to optimize the statistical quality 


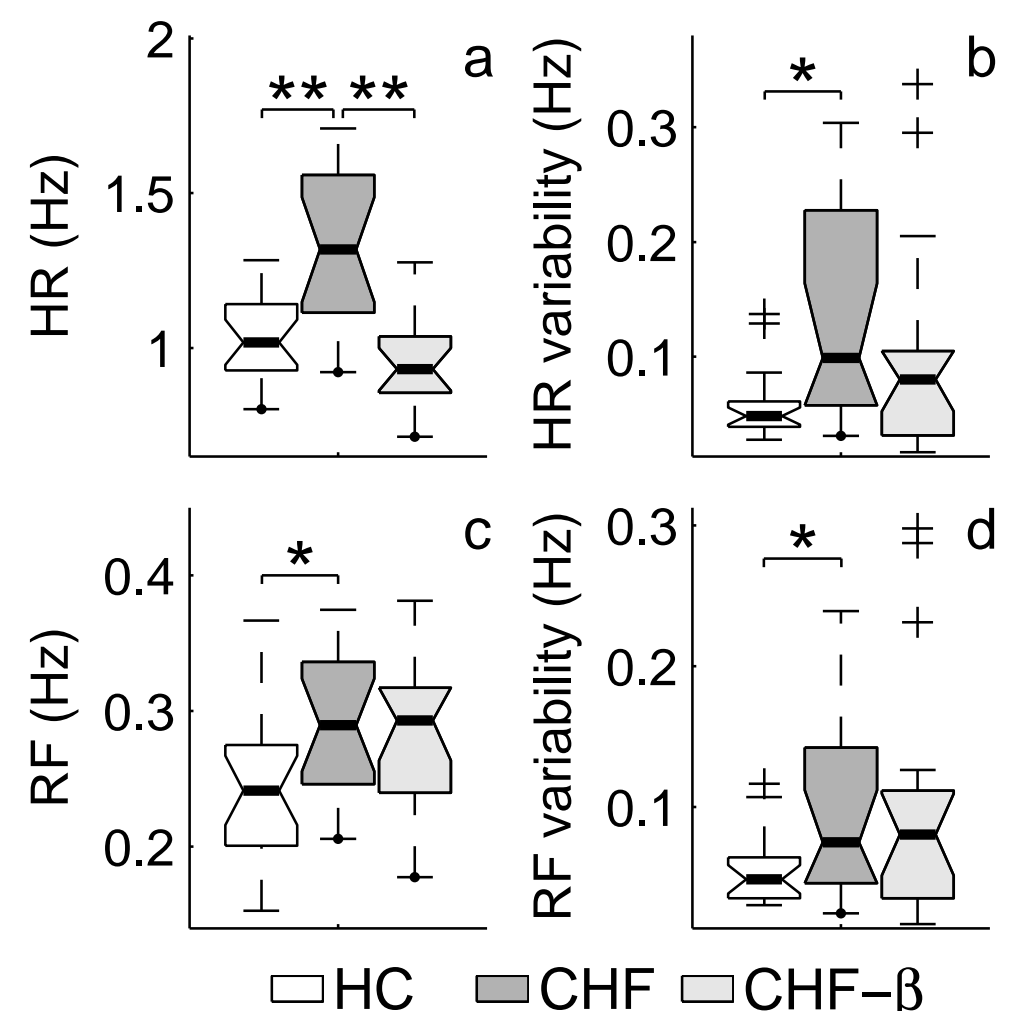

Figure 2. Summary of heart and respiration characteristics for the three subject groups. a, Heart rate; b, heart rate variability; c, respiratory rate, d, respiratory rate variability.

of the analyses, and the obvious practical limitations in terms of how long a subject can be expected to stay still and thus not introduce movement artifacts into the data.

Skin blood flow signals were measured by the laser Doppler perfusion technique. Two channels of a Moor Instruments Ltd (Axminster, UK) DRT4 LDF monitor were used in conjunction with probes (Moor MPI-V2) mounted within iontophoresis chambers (Moor MIC1-IONlr). Measurements were made on the volar aspect of the right forearm, choosing areas of skin that were free from blemishes. To avoid possible systematic bias, we attempted to position both probes in areas with similar vasculature and without any larger vessels visible in their vicinity; the two chambers were placed randomly relative to each other for each subject, and for every recording. Difference in response to ACh and SNP found in the two lowest frequency intervals (see below) are therefore most unlikely to result from chance; the difference at $0.01 \mathrm{~Hz}$ had already been consistently demonstrated in several study groups of different age. ${ }^{1,11,13}$ Near-infra-red light (wavelength $780 \mathrm{~nm}$ ) was delivered to the probes via a nearly loss-free optical fibre. The back-scattered light was collected and returned to the floLab monitor via another optical fibre, where it was converted to an analogue electrical signal. The cut-off frequency of the low-pass filter was $22.5 \mathrm{kHz}$, and an output time constant of $0.1 \mathrm{~s}$ was selected. Signals were digitized at $400 \mathrm{~Hz}$ with 16-bit resolution by use of a National Instruments PCI-6035E A/D converter, and stored in a personal computer. The LDF probes were calibrated against the flux standard provided by Moor Instruments in order to allow comparison of different signals and the blood flow was expressed in arbitrary units (AU).

The two vasodilators differ in that the action of ACh is endothelium-dependent whereas that of SNP, a direct NO donor, is endothelium-independent. The difference in the blood flow enhancements that they produce in any oscillatory component is thus related to the responsiveness of the vascular endothelium. ${ }^{11-13}$ The $1 \%$ ACh and SNP solutions were prepared on the day of the study in each case. They were drawn through the cutaneous barrier at each of the LDF measuring sites by means of a constant electrical current of $100 \mu \mathrm{A}$ derived from a 
Moor Instruments MIC1-e current controller. The iontophoresis current was applied in 7 pulses of $20 \mathrm{~s}$, with a separation of $240 \mathrm{~s}$ between each, as indicated in Figure 1a. Examples of blood flow changes for a healthy subject in response to the ACh and SNP are shown in Figure 1b,c, illustrating the large increase of oscillatory activity that typically occurs in addition to the increased average flow.

\section{DATA ANALYSIS}

The time averages of the signals from the LDF probes (originally sampled at $400 \mathrm{~Hz}$ ) were calculated, and the signals were then resampled at $10 \mathrm{~Hz}$ using a moving average technique. Trends were removed by giving the moving average a window length of $200 \mathrm{~s}$, thereby eliminating frequencies below $0.005 \mathrm{~Hz}$. Following this preprocessing, we applied the wavelet transform (using the Morlet mother wavelet) to analyse the frequency content of the signals. The advantage compared to conventional Fourier techniques is the logarithmic abscissa, enabling an extremely wide range of frequencies to be accommodated: in the present case, the characteristic frequencies differed by a factor of more than $100 \times$. In analysing the data, we have found evidence for activity in a frequency interval lower than those previously reported, centred on $0.007 \mathrm{~Hz}$. In what follows, we will refer to the latter as the "lowest frequency interval".

We use two measures to quantify the contribution of the oscillations to the total blood flow: their average amplitude, calculated over the whole frequency interval and within the subintervals; and the normalized amplitude, defined as the ratio between the average amplitude within a given subinterval and the average amplitude over the whole frequency interval $(0.005-2.00 \mathrm{~Hz})$. A nonpaired, nonparametric, Wilcoxon rank sum test was used to obtain the probability that two distributions are equal. The value $p<0.05$ was considered to imply a statistically significant difference. The graphical data that follow below are presented in each case as a box with lines drawn at the 10th pencentile, the median and the 90th percentile. Whiskers extend from the ends of the box to the most extreme data value within 1.5 $\times$ IQR (where IQR is the inter-quartile range of the box). Outlying data falling beyond the whiskers' range are marked with a + . Pairs of distributions with $0.01<p<0.05$ are asterisked; where $p<0.01$ a double-asterisk is used.

\section{RESULTS}

Figure 2 summarises the heart and respiration statistics for the three subject groups. In the CHF group, heart rate (HR) and heart rate variability (HRV) are significantly increased as compared to the age-matched HCs. The increased HRV is mainly on account of ectopic beats. $\beta$-blockers evidently cause a significant decrease in HR but not in HRV. The respiration frequency (RF) was defined as the reciprocal of the interval between successive respiration maxima, averaged over 30 minutes. Both $\mathrm{RF}$ and respiration frequency variability (RFV) were significantly increased in CHF patients as compared to the HC group. Neither RR nor RFV were significantly affected by the treatment with $\beta$-blockers.

Figure 3a shows the effect of ACh and SNP on the mean flows in HC and CHF groups, and Figure 3b shows their effect on the total spectal amplitude. There are highly significant differences in the mean flows between the two subject groups, in their responses to both ACh and SNP. However, there are no statistically significant differences between them in the total spectral amplitudes of their responses to ACh and SNP.

With added frequency discrimination, it becomes evident that almost all the oscillatory components are affected by CHF, but that the impairments of the lower frequency ones in CHF patients are only seen with ACh (and not with SNP). There is a clear distinction between the CHF and HC groups in their spectral amplitudes in response to $\mathrm{ACh}$ in the lowest frequency interval, but not in response to SNP. The same is true of their normalised amplitudes.

The effect of $\beta$-blockers has been analysed, comparing the responses to ACh and SNP for all three subject groups. The most significant comparisons are again in the lowest frequency interval. Here, there is a highly significant difference in the responses to ACh and SNP on the part of the HC group in terms of both spectral amplitude and normalised amplitude. There is no statistically significant difference for spectral amplitude in the case of the CHF patients, but there is a significant difference in their normalised amplitudes. Following treatment with $\beta$-blockers, however, the CHF- $\beta$ group exhibits differences in response to ACh and SNP that are statistically highly significant, both in the spectral amplitude and in the normalised amplitude. 


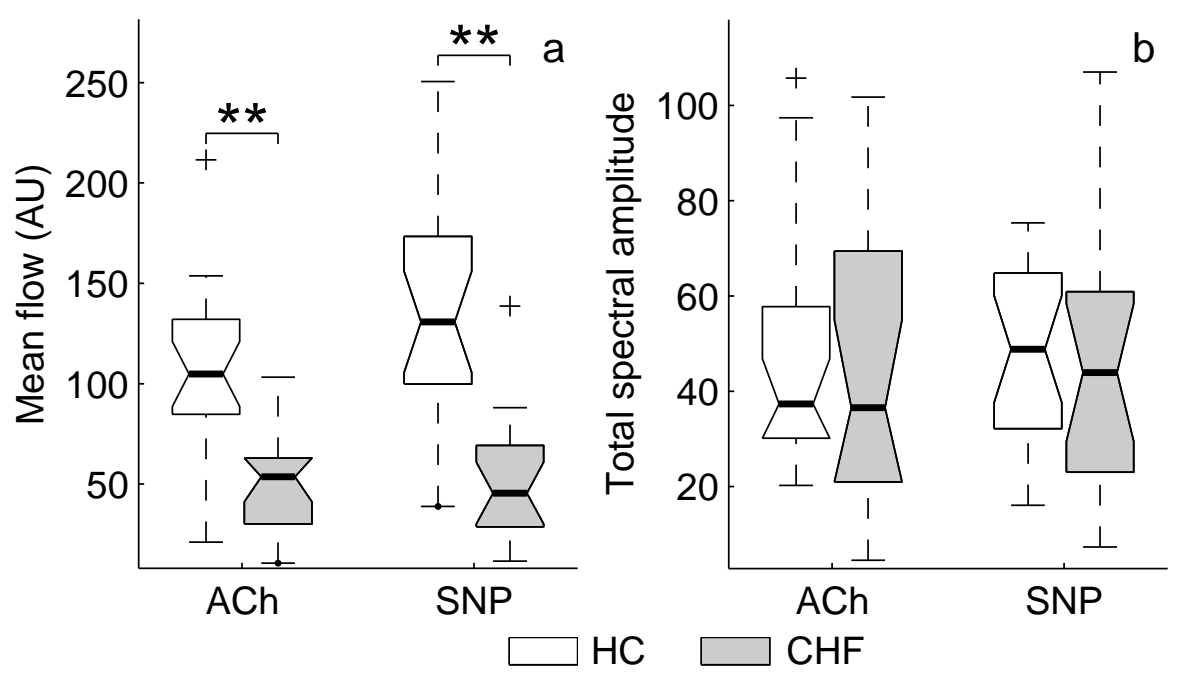

Figure 3. The effects of ACh and SNP on the mean blood flow and total spectral amplitude for the CHF and HC subject groups.

\section{DISCUSSION AND CONCLUSIONS}

The fact that the lowest frequency peak is affected by ACh but not SNP in the HC group can be taken as evidence that, like the peak at $0.01 \mathrm{~Hz}$, it is associated with endothelial function. Our results clearly demonstrate that the responsiveness of the vascular endthelium in CHF patients is reduced, as compared to the HC group. This finding is consistent with the recent observation ${ }^{10}$ by Morgan et al of decreased endothelial responsiveness in the larger vessels of CHF patients. The latter authors were also investigating elderly patients, and also using ACh and SNP. Their study involved invasive techniques for the measurement of average flows, without the frequency discrimination possible in studies of oscillations. All of the patients were receiving medication, so there was no opportunity to study changes resulting from treatment.

The differing responses of the CHF and CHF- $\beta$ groups found in the present study show clear evidence that treatment with Bisoprolol brings an increase in endothelial responsiveness in the lowest frequency interval: the ACh/SNP differentiation in spectral amplitude for untreated CHF patients is statistically insignificant whereas, for the CHF- $\beta$ group it is statistically highly significant. A similar pattern is evident for changes in the normalised amplitude in the lowest frequency interval, where a statistically significant ACh/SNP differentiation in the CHF group becomes highly statistically significant in the CHF- $\beta$ group following treatment. Treatment with Bisoprolol evidently ameliorates the impairment of endothelial responsiveness associated with CHF. There is clear potential for routine clinical use of LDF, coupled with iontophoretic delivery of ACh and SNP and frequency analysis, in the assessment of a CHF patient's endothelial reactivity, and for quantification of the extent to which a more normal response can be restored by treatment with Bisoprolol.

A full account of this work, together with the detailed frequency analyses of the results, will be presented elsewhere.

\section{ACKNOWLEDGMENTS}

We gratefully acknowledge the assistance of Alexander Balanov, Natalia Janson, Mark Lippett and Yuri Shiogai in recording the data. The research was supported in part by the Slovenian Ministry of Education, Science and Sport, by the Engineering and Physical Sciences Research Council (UK), by the Medical Research Council (UK), by the Leverhulme Trust (UK) and by the Wellcome Trust (UK). 


\section{REFERENCES}

1. A. Stefanovska and M. Bračič, "Physics of the human cardiovascular system," Contemporary Phys. 40(1), pp. 31-55, 1999.

2. H. D. Kvernmo, A. Stefanovska, K.-A. Kirkebøen, and K. Kvernebo, "Oscillations in the human cutaneous blood perfusion signal modified by endothelium-dependent and endothelium-independent vasodilators," $M i$ crovasc. Res. 57(3), pp. 298-309, 1999.

3. E. J. Eichhorn and M. R. Bristow, "Medical therapy can improve the biological properties of the chronically failing heart - A new era in the treatment of heart failure," Circulation 94(9), pp. 2285-2296, 1996.

4. M. R. Bristow, " $\beta$-adrenergic receptor blockade in chronic heart failure," Circulation 101, pp. 558-569, 2000 .

5. J. López-Sendón and et al, "Expert consensus document on $\beta$-adrenergic receptor blockers," European Heart J. 25, pp. 1341-1362, 2004.

6. H. Drexler, "Effect of angiotensin-converting enzyme inhibitors on the peripheral circulation in heart failure," Am. J. Cardiol. 70, pp. 50C-54C, 1992.

7. C. V. Leier, "Regional blood flow in human congestive heart failure," Am. Heart J. 124, pp. 726-738, 1992.

8. H. Drexler, D. Hayoz, T. Munzel, and et al, "Endothelial function in chronic congestive heart failure," Am. J. Cardiol. 69, pp. 1596-1601, 1992.

9. P. M. Vanhoutte, "Endothelium-dependent responses in congestive heart failure," J. Mol. Cell. Cardiol. 28, pp. 2233-2240, 1996.

10. D. R. Morgan, L. J. Dixon, C. G. Hanratty, S. M. T. Hughes, W. J. Leahey, K. P. Rooney, C. D. Johnston, and G. R. McVeigh, "Impaired endothelium-dependent and -independent vasodilation in elderly patients with chronic heart failure," European J. of Heart Failure 6, pp. 901-908, 2004.

11. H. D. Kvernmo, A. Stefanovska, K. A. Kirkebøen, and K. Kvernebo, "Oscillations in the human cutaneous blood perfusion signal modified by endothelium-dependent and endothelium-independent vasodilators," $M i$ crovasc. Res. 57(3), pp. 298-309, 1999.

12. A. Stefanovska, M. Bračič, and H. D. Kvernmo, "Wavelet analysis of oscillations in the peripheral blood circulation measured by laser Doppler technique," IEEE Trans. Bio. Med. Eng. 46(10), pp. 1230-1239, 1999.

13. P. Kvandal, A. Stefanovska, M. Veber, H. D. Kvernmo, and K.-A. Kirkebøen, "Regulation of human cutaneous circulation evaluated by laser doppler flowmetry, iontophoresis, and spectral analysis: importance of nitric oxide and prostaglandines," Microvasc. Res. 65(3), pp. 160-171, 2003. 